\title{
Methodology for controller interaction assessment in distribution networks with a high share of renewable energy
}

\author{
Jorge Velasquez \\ From The 7th DACH+ Conference on Energy Informatics \\ Oldenburg, Germany. 11-12 October 2018
}

\section{Correspondence:}

jorge.velasquez@offis.de OFFIS Institute for Information Technology, Escherweg 2, 26121 Oldenburg, Germany

\begin{abstract}
The roll-out of decentralized energy resources introduces a set of new challenges for system operators. In order to address these requirements it is necessary to utilize modern control strategies, and consequently the use of automation and Information and Communication Technologies. Nevertheless, the use of multiple control schemes in a complex grid may result in a conflicting behavior of two or more control devices which ultimately lead to an undesired behavior of the controlled power plants. Nowadays, there are several efforts toward the early identification and mitigation of controller conflicts within complex energy systems. However, a comprehensive methodology to address the dynamics of the system from a broader perspective is not yet fully established. For this reason, this research project presents a method for identifying and testing potential controller conflicts considering the dynamic behavior of the components within the system under study by using multiple models and analysis tools as a part of a toolchain integration approach.
\end{abstract}

Keywords: Power system simulation, Renewable energy sources, Dynamic security assessment

\section{Motivation}

Traditionally, ancillary services are provided by the large scale utilities following the requirements given by the Transmission System Operator (TSO). However, the decommissioning of large scale power plants in Germany requires that renewable energies (i.e. large scale wind and PV) are able to cope with such obligations to ensure the stability of the grid (Eurelectric 2004). For this reason, there is an increasing need for system operators to innovate in order to further improve the monitoring and management of the electrical grid (Eurelectric 2016). One way to deal with this challenge is the use of Information and Communication Technologies (ICT), this provides the opportunity to automate and control the network in an optimized manner. However, it also adds complexity and poses several barriers for the systems operators to overcome, especially for Distribution System Operators (DSO) that are used to operate under reliable condition at the medium and low voltage level. However, it is likely this situation may result in a conflicting behavior as a consequence in the use of multiple

(c) The Author(s). 2018 Open Access This article is distributed under the terms of the Creative Commons Attribution 4.0 International License (http://creativecommons.org/licenses/by/4.0/), which permits unrestricted use, distribution, and reproduction in any medium, provided you give appropriate credit to the original author(s) and the source, provide a link to the Creative Commons license, and indicate if changes were made. 
new and untested control schemes in the electrical grid. In other words, it is possible that two or more control strategies collide when there are several global and local optimization objectives. This undesired behavior could derive into operational inefficiencies at best or major stability problems at worst.

Nowadays, there are several efforts towards the management of controller conflicts in electrical power systems. However, there is still potential for a method that is able to systematically test the behavior of multiple controllers in a dynamic manner. With this in mind, the goal of the proposed approach is to assess if the interaction of a set of controllers might hamper the safety operation of a particular distribution grid. Moreover, the research artifact is divided into two parts. The initial step is the preliminary analysis of the system and the mapping of the interaction of the main components under study from a requirement engineering perspective. Secondly, is the dynamic simulation and the systematic testing of controllers interactions. Important to realize is that the main goal of this approach is to early identify and test potential negative interactions of controllers focusing on distribution grids with a high share of renewable energies. Thus the main outcome of this research is the reliable operation of distribution grids, which implies that this method strives to perform as fast as possible meaning near (e.g. $15 \mathrm{~min}$ ) to the real operation of the grid.

\section{Preliminary results}

As mentioned above, electrical power systems are undergoing important transformations with the increased adoption of decentralized energy resources. This changes introduce, among others, major sources of uncertainties in the management and operation of future electrical grids. Consequently, one research question is how to systematically test controller interactions to determine if there is a negative behavior that can hinder the operation of distribution grids. With this in mind, OFFIS has been focused on addressing this question in the project EWE Netz Dynamische Simulation.

In the context of this research project several control are used for either voltage or reactive power support within the grid. They are on-load tap changer of the main transformer and inverter based control for renewable generators $(\cos \phi(P)$ and $Q(U)$ characteristics) as well as reactive power control for wind farms $[\mathrm{Q}(\mathrm{Q})$ characteristic].

In the following, one of the most prominent interaction is presented. In this particular case three control strategies are operating at the same time. First is the tap changer control for the main transformer with a dynamic set-point adjustment (this configuration is defined to adjust the voltage increase that ensues after a sudden influx of active power through the transformer). Second is the reactive power control for two wind farms connected at the main busbar i.e. $\mathrm{Q}(\mathrm{Q})$ control. The last one is $\cos \varphi(\mathrm{P})$-characteristic for the remaining wind farm located far away from the main busbar. In the Fig. 1 the main results are presented.

As mentioned before the use case for this project is voltage and reactive power control. In the figure, the medium voltage at the main busbar at $20 \mathrm{kV}$ is shown. Additionally it is shown the reactive power provision from the onshore wind farms. The closest one to the main busbar is providing reactive power for attempting to fulfill the grid operator requirement of $\mathrm{Q}=0$ at the point of common coupling (interconnection with the external grid). Furthermore, the remaining wind farms within the distribution grid 


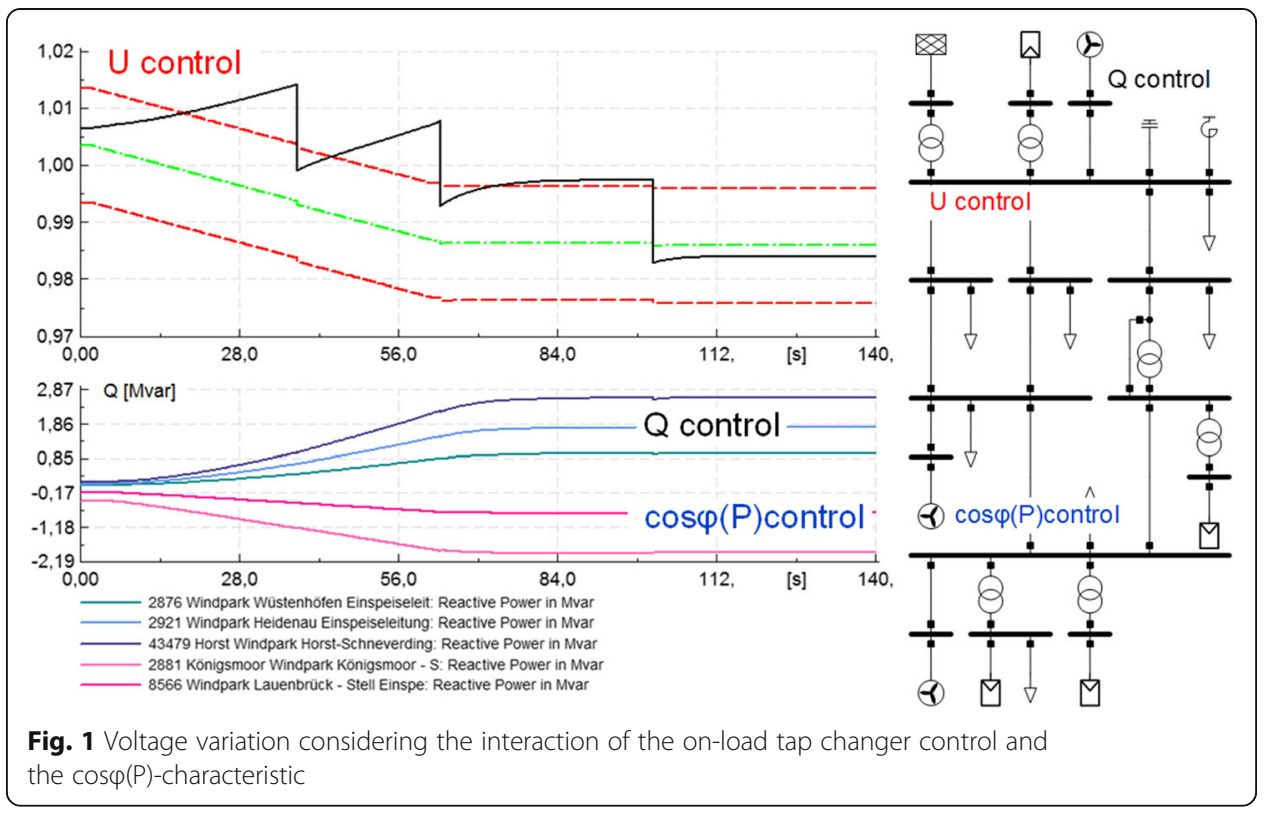

are set to control the voltage via providing or absorbing reactive power in the so called $\cos \phi(\mathrm{P})$ control for decentralized energy resources.

The interaction of the three control strategies shown in Fig. 1 is considered an operational inefficiency because while the medium voltage is on the rise due to a sudden feed-in of active power from the PV units in the systems the wind farm within the grid should absorb reactive power in order to provide voltage support thus decreasing its magnitude. However, around the same time there is a requirement to keep the reactive power balance at the point of common coupling which result in a reactive power provision coming from the closest wind farm connected to the $20 \mathrm{kV}$ busbar. Consequently, both the absorption and production of reactive power from two different wind farms are canceling each other which results in an uncontrolled rise of the voltage. This is not considered a critical problem because the tap changer control of the transformer is able to act thus bringing the voltage value back to the defined deadband. Important to realize however is that the voltage is adjusted after three tap action in a time frame of less than $2 \mathrm{~min}$, this is also undesired when considering the wear and tear of the mechanical parts of the tap changer of the main transformer.

In essence, the aforementioned project is relevant to the current research because it addresses both the requirement engineering process (points 1 through 3 in the Fig. 2) and the dynamic testing of controller interactions (point 4). However, there are still open points to deal in the context of this research. Firstly is that there has been no requirement for the use of a formal use case elicitation (chiefly the use of the IEC 62559-2) and its mapping onto the SGAM as opposed to this research project. This entails that it is important to describe the main use case derived from this project according to the presented artifact. Secondly, it is necessary to use, during the testing phase, a more general design to increase the robustness the proposed approach (e.g. developing a set of synthetic grid models that consider different topologies). Additionally, another important aspect is the definition of an appropriate evaluation method for the optimization of the simulation analysis based on modern design of experiments 


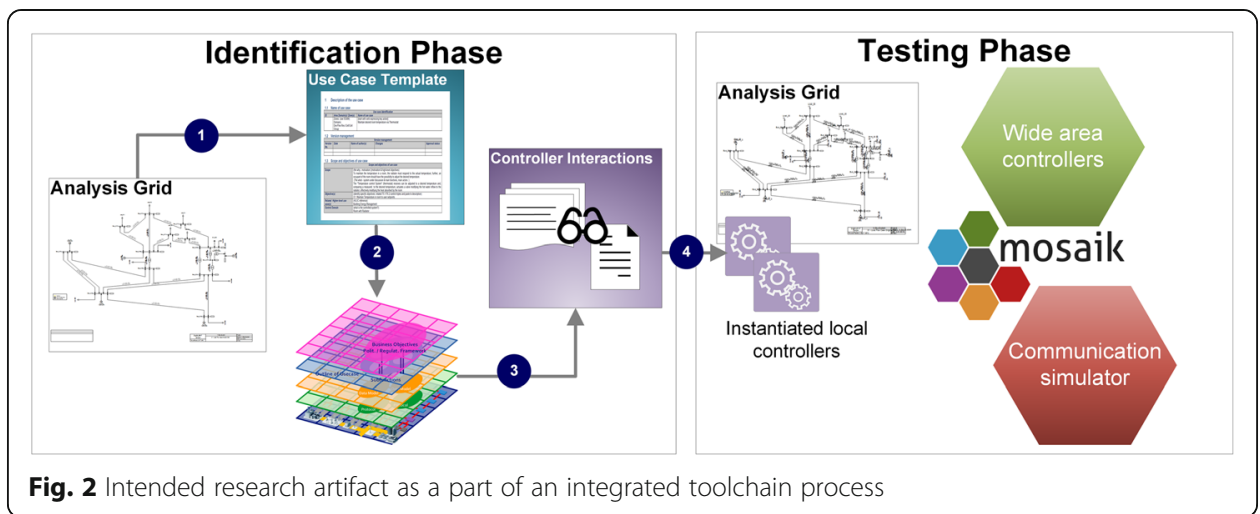

techniques allowing a comprehensive interaction analysis considering a complex combinatorial problem with increased time constraints taking into account that this method should be able to perform as close as possible to the operation of the power system.

\section{Methodology}

Early detection of potential controller conflicts is one important factor when considering the adoption of decentralized resources as well as innovative and untested control strategies. Equally important is to gather the requirements across multiple domains and evaluate the potential impact of the interaction of heterogeneous control architectures for different grid topologies, operational scenarios and events. As a result the initial phase of this research is based upon existing tools that utilize a standard method for use case description and management (based on the IEC 62559-2). This first step is presented in the Fig. 2.The objective of this step is to list the main actors of the system and their respective goals. Another important aspect at this stage is the definition of the operational scenarios (e.g. high in-feed from decentralized energy resources and low demand) associated to the main use case to realize. In addition, a list of network events is required to trigger a control response (e.g. switch action, voltage dips, DER ramps) thus enabling the study of interactions of controllers.

The second step as shown in the figure is the mapping of the use case in SGAM in order to asses, in a visual manner, the interactions and interdependencies across all the interoperability layers defined in the architectural model (mainly the component, communication and information layer). This allows the possibility to identify potential undesired interactions at an early stage of the analysis. A final step is defined (third point in the figure) and it is the listing of possible controller combinations based on the information collected on the use case template. In addition, this list of combinations can be further evaluated to optimize the search space (mainly reducing and avoiding unnecessary controller evaluations) thus conserving computational expensive resources (Castro 2018).

Finally, the list of possible controller interactions is systematically tested (marked in the figure as point 4). Important to realize is that the combination of the static analysis (during the identification phase) is complemented with a co-simulation framework as a part of a toolchain integration. Consequently, several analysis tools are used to conceive a specific use case. For this reason, mosaik is a central component in the testing of controller interactions. This co-simulation framework allows the orchestration of power 
system analysis tools, for the evaluation of the grid dynamics, and a communication simulator to integrate the effect of poor quality of service for the associated ICT elements (e.g. when considering a combination of wide area and decentralized control).

The systematic testing of controller interactions is performed in a two-step process. First is the definition of the possible combinations depending on the type of control strategies available for a specific use case. At this stage it is important however, to delimit the complex combinatorial problem this entails, considering a large amount of controller throughout the grid with multiple control modes available at hand. One alternative is to enumerate the possible combinations and to cluster them according to a set of criteria (i.e. critical for the operation, likely to occur or similarity among others). Secondly, once the most prominent combinations are defined it is possible to test how the parametrization of the controllers under study might hamper the stability of the system. In this case, two or more parameters are modified according to the type of controller for a given combination and tested for potential conflicts. However, at this stage it is important to optimize the high computational cost that entails the simulation of a high amount of possibilities given the system requirements. One possibility is the use of sampling based methods rooted in modern design of experiments (DoE) to explore the search space in an efficient manner.

\section{Conclusion}

The proposed method aims to first describe and formalize the different use cases which can produce a controller conflict based on known assumption of the electrical grid under study. However, a static analysis of the system is not enough to prove whether there is a potential issue in the system (e.g. stability problems or inefficiencies). Correspondingly, another aspect is the use of coordinated tools to test the interactions of the controller and the elements within the power grid in a dynamic manner. For this reason, it is important to determine the different acting components (e.g. distribution transformers, loads, generators) and its interaction with the control devices under study. Additionally, it is also relevant to define what the variables to control are. This description is helpful to identify how the objectives of the evaluated controllers may conflict within a specific grid.

Equally important step is the testing and verification process of the possible conflicting behavior between multiple control strategies. Correspondingly, co-simulation schemes are employed to model the behavior of the different components interacting in a given grid topology. Additionally, a mitigation strategy has to be implemented to reduce or eliminate the source of the conflict. At this stage it is necessary to provide a series of recommendations to address potential issues that might hinder the power system operation.

In summary, at the current stage of this research there is already an existing approach for testing controller interactions. However, additional effort are required for the requirements elicitation considering the main stakeholders and use cases. Furthermore, it is important to develop a suitable evaluation method that is able to survey the defined search space in an efficient manner. 


\section{Acknowledgements}

I would like to express my sincere gratitude to my advisor Prof. Dr. Sebastian Lehnhoff for the continuous support and helpful insights of my PhD study and related research. In addition, I would like to thank Dr. Friederich Kupzog from AlT for his help in the improvement of the contents of the paper as part of the shepherding process of the Energy Informatics PhD workshop 2018.

Funding

Publication costs for this article were sponsored by the Smart Energy Showcases - Digital Agenda for the Energy Transition (SINTEG) program.

\section{Availability of data and materials}

Not applicable

\section{About this supplement}

This article has been published as part of Energy Informatics Volume 1 Supplement 1, 2018: Proceedings of the 7th DACH+ Conference on Energy Informatics. The full contents of the supplement are available online at https://energyinformatics.springeropen.com/articles/supplements/volume-1-supplement-1.

\section{Author's contributions}

$J V$ prepared and analyzed all the data presented in this research paper. The author read and approved the final manuscript.

\section{Competing interests}

The author declares that he has no competing interests.

\section{Publisher's Note}

Springer Nature remains neutral with regard to jurisdictional claims in published maps and institutional affiliations.

Published: 10 October 2018

\section{References}

Castro F (2018) Intelligent assessment of dynamic security in distribution networks with high penetration levels of intermittent decentralized generating units

Eurelectric (2004) Ancillary services unbundling electricity products - an emerging market

Eurelectric (2016) Innovation incentives for DSOs - a must in the new energy market development

\section{Submit your manuscript to a SpringerOpen ${ }^{\circ}$ journal and benefit from:}

- Convenient online submission

- Rigorous peer review

- Open access: articles freely available online

High visibility within the field

- Retaining the copyright to your article 